\title{
PREDICTIVE MODELING OF PERI-OPERATIVE MORBIDITY
}

\author{
Peter C MacDougall PhD MD, Stephen D Beed MD, Paul A Brousseau BEd, Caren L Rose MSc \\ Department of Anesthesia, Dalhousie University, Queen Elizabeth II Health Sciences Centre, \\ 1796 Summer Street, Halifax, NS, B3H 2A7
}

\section{INTRODUCTION}

Early identification of patients who may require an extended length of stay (LOS) or ICU care is important for health care resource planning. We use a novel modeling method, Classification and Regression Trees (CART), to develop a predictive model of peri-operative morbidity following inpatient larparoscopic cholecystectomy.

\section{METHODS}

After obtaining approval from the Research Ethics Board, a retrospective chart review of all inpatients having laparoscopic cholecystectomy over a 20 month period was carried out. Data were collected on multiple co-morbid conditions. Classification and Regression Trees ${ }^{1}$ were created using S-Plus 6.1 for hospital length of stay (LOS) and ICU admission. Ten fold internal cross validation was performed on the results. Linear and logistic regression analyses were carried out on the same data sets for comparison.

\section{RESULTS}

Using a classification tree with ICU admission as a response demonstrated that only patients > 45.5 years were admitted to ICU. Predictors of ICU admission were ischemic heart disease (IHD) plus open cholecystectomy and age greater than 73.5 years. Logistic regression identified age, IHD, surgical complications, chronic obstructive pulmonary disease, and open surgery as predictors of ICU admission. Regression trees were created with LOS as a response both with and without ICU admission as a variable. When ICU admission was included as a variable, dichotomization occurred initially at ICU admission. In patients admitted to ICU $(n=16)$ the most significant predictors of LOS were age and body mass index (BMI). Of patients not admitted to ICU $(n=277)$ the most significant predictors of LOS were open cholecystectomy, BMI, increased age, emergent surgery, and dysrhythmias. Linear regression analysis of the same data demonstrated that age, emergent surgery, open cholecystectomy and surgical complications were predictive of increased LOS. Excluding ICU as a predictor of LOS demonstrated that age, sex, BMI, dysrhythmias and IHD were predictive.

\section{DISCUSSION}

CART (1) is a useful method of predicting peri-operative morbidity and resource utilization. Predictors of peri-operative morbidity identified by CART were similar to those identified by linear and logistic regression techniques. Further, CART demonstrates the interaction between predictive variables.

\section{REFERENCES}

Classification and Regression Trees. Wadsworth, Belmont Ca. 1984 